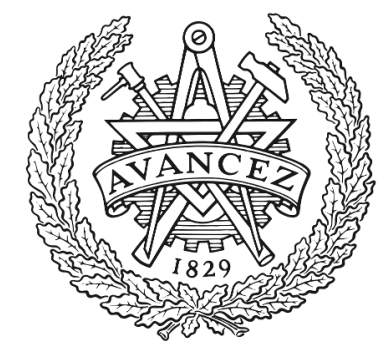

\title{
CHALMERS
}

UNIVERSITY OF TECHNOLOGY

\section{Stepped-Carrier OFDM V2V Resource Allocation for Sensing and Communication Convergence}

Downloaded from: https://research.chalmers.se, 2023-04-26 08:26 UTC

Citation for the original published paper (version of record):

Keskin, F., Aydogdu, C., Wymeersch, H. (2020). Stepped-Carrier OFDM V2V Resource Allocation for Sensing and Communication Convergence. 14th European Conference on Antennas and Propagation, EuCAP 2020. http://dx.doi.org/10.23919/EuCAP48036.2020.9135203

N.B. When citing this work, cite the original published paper. 


\title{
Stepped-Carrier OFDM V2V Resource Allocation for Sensing and Communication Convergence
}

\author{
Musa Furkan Keskin, Canan Aydogdu, Henk Wymeersch \\ Department of Electrical Engineering, Chalmers University of Technology, Gothenburg, Sweden. \\ e-mail: furkan@chalmers.se.
}

\begin{abstract}
Stepped-carrier orthogonal frequency division multiplexing (OFDM) radar is a promising low-cost alternative to conventional OFDM radar for automotive applications due to its capability to provide high resolution with low-rate analogto-digital converters (ADCs). In this paper, we investigate centralized time-frequency resource allocation strategies in vehicular networks for vehicle-to-vehicle (V2V) sidelinks employing stepped-carrier OFDM waveform for joint radar sensing and communications. To quantify radar-communication performance trade-offs, we formulate a nonlinear integer programming problem for weighted optimization of radar accuracy and communication spectral efficiency, and perform Boolean relaxation to obtain an efficiently solvable convex program. Simulation results demonstrate radar-optimal and communication-optimal operation regimes, providing insights into time-frequency weightings along the trade-off curve.
\end{abstract}

Index Terms-orthogonal frequency division multiplexing, joint radar communications, resource allocation.

\section{INTRODUCTION}

Co-design of radar and communication systems has recently received a growing interest as mutual interference becomes a compelling issue in view of a large number of spectrally co-existent radars and communication devices [1]. This trend manifests itself especially in the automotive applications, where a rising percentage of vehicles is equipped with sensors and vehicle-to-vehicle (V2V) radio technology for autonomous driving functionalities [2], [3].

To tackle the problem of spectral congestion in vehicular scenarios, a popular co-design approach is to employ orthogonal frequency-division multiplexing (OFDM) as a dualfunctional radar-communication (DFRC) waveform as it allows for easy interaction with communication, while still providing satisfactory radar performance [4]-[8]. The study in [4] proposes an algorithm for target range and velocity estimation using the backscattered OFDM signal. In a similar fashion, [5] addresses the design of an OFDM system where pilots are used by radar receiver for target parameter estimation and by communication receiver for channel estimation. Another research strand focuses on OFDM DFRC waveform design via joint optimization of radar and communication performance objectives [6]-[8]. Mutual information (MI) has been used to characterize both radar and communication performance [6], [7], while the Cramér-Rao bound (CRB) is a widely employed metric for radar estimation accuracy [8].

In this paper, we consider a vehicular joint radarcommunications network in which a base station (BS) cen- trally coordinates time-frequency V2V sidelink resource sharing among vehicles through uplink/downlink communications. The available time-frequency window for sidelink consists of individual OFDM blocks, each containing a number of subcarriers and symbols, as shown in Fig. 1. Unlike the OFDM DFRC frequency domain waveform design studies [4]-[8], our problem of interest herein is to assign OFDM resource blocks to vehicles in the time-frequency domain to maximize overall radar communications performance of the network (which leads to stepped-carrier OFDM [9] radar due to frequency hopping across blocks). Using Boolean resource selection parameters of vehicles as variables, a nonlinear integer programming problem is formulated to optimize a compound objective function as a weighted combination of radar $\mathrm{CRB}$ and communication spectral efficiency (SE). Through a Boolean relaxation, we transform the optimization problem into an efficiently solvable convex semidefinite program (SDP). Simulation results provide valuable insights into delay-Doppler and radar-communication performance tradeoffs.

\section{System MODEL}

We consider an OFDM system with a collection of $N$ vehicles and a base station (BS), as shown in Fig. 1. A total bandwidth $W$ is divided into parts: $W_{\mathrm{UD}}$ for uplink and downlink data traffic as well as control signaling, and $W_{\mathrm{S}}$ for sidelink traffic. The ADC bandwidth at each vehicle is $W_{\text {block }} \ll W_{\mathrm{S}}$. The OFDM system uses a subcarrier spacing $\Delta_{f}=1 / T$, with the number of subcarriers $K=W_{\text {block }} / \Delta_{f}$, and has a basic OFDM symbol duration $T_{\mathrm{s}}=T+T_{\mathrm{cp}}$. In a frame-time of $T_{\text {frame }}$, we have a total of $W_{\mathrm{S}} / W_{\text {block }} \times T_{\text {frame }} / T_{\text {block }}$ timefrequency resource blocks, where each block comprises $M$ OFDM symbols and has a total duration $T_{\text {block }}=M T_{\mathrm{s}}$.

Our goal is to assign resources $b_{i_{f}, i_{t}}^{(n)} \in\{0,1\}$ to each vehicle $n \in \mathcal{N}$ to jointly optimize data rate and radar accuracy, where $i_{f} \in \mathcal{F} \triangleq\left\{0,1, \ldots, W_{\mathrm{S}} / W_{\text {block }}-1\right\}$, $i_{t} \in \mathcal{T} \triangleq\left\{0,1, \ldots, T_{\text {frame }} / T_{\text {block }}-1\right\}$ and $\mathcal{N}$ is the set of vehicles in the network. To ensure orthogonality among users, we enforce $b_{i_{f}, i_{t}}^{(n)} \times b_{i_{f}, i_{t}}^{\left(n^{\prime}\right)}=0$ for any two users $n$ and $n^{\prime} \neq n$. In addition, due to the ADC bandwidth constraint, each vehicle can occupy at most one resource block at a given time, i.e., $b_{i_{f}, i_{t}}^{(n)} \times b_{i_{f}^{\prime}, i_{t}}^{(n)}=0$ for $i_{f} \neq i_{f}^{\prime}$.

\section{Communication Performance Metric}

We consider unicast communication, where for each vehicle $n \in \mathcal{N}$, there is a dedicated receiver $\mathcal{R}(n) \in \mathcal{N}$. We 


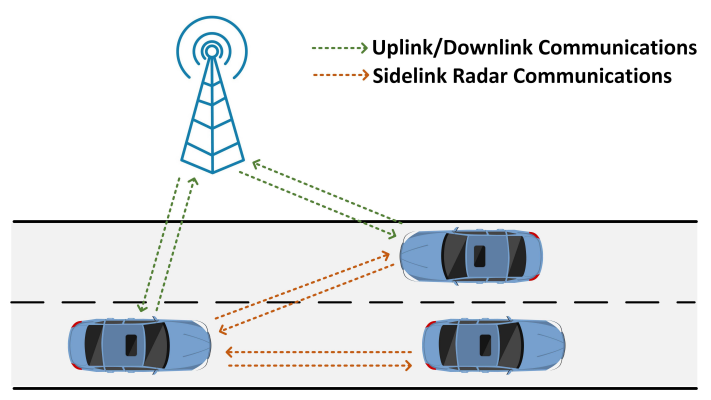

(a)

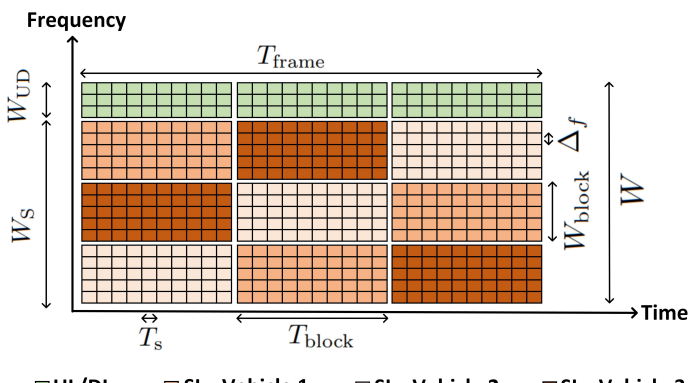

(b)

Fig. 1. (a) A vehicular radar communications network whose time-frequency resource allocation over sidelink is coordinated by a base station through uplink/downlink communications. (b) Exemplary time-frequency resource sharing among three vehicles over sidelink, where the aim is to perform joint optimization of overall data rate and radar accuracy.

assume that the receiver knows instantaneous channel state information (CSI) and both the transmitter and the receiver have statistical CSI (which remains constant over a frame duration). The $k$ th OFDM subchannel gain corresponding to the transmit-receive pair $(n, \mathcal{R}(n))$ for the frequency block $i_{f}$ is assumed to be zero-mean complex Gaussian random variable with variance $\left(\sigma_{i_{f}}^{(n, \mathcal{R}(n))}[k]\right)^{2}$ for $k=0, \ldots, K-1$, known to the BS through uplink. In this scenario, the performance can be measured in terms of sum SE over the blocks, which can be upper-bounded as

$$
R^{(n)} \leq \sum_{\left(i_{f}, i_{t}\right) \in \mathcal{F} \times \mathcal{T}} b_{i_{f}, i_{t}}^{(n)} R_{i_{f}, i_{t}}^{(n)}
$$

where the upper bound for individual blocks is given by [10, Ch. 4.3.3]

$$
R_{i_{f}, i_{t}}^{(n)}=\sum_{k=0}^{K-1} \log _{2}\left(1+\frac{P_{i_{f}, i_{t}}^{(n)}[k]\left(\sigma_{i_{f}}^{(n, \mathcal{R}(n))}[k]\right)^{2}}{N_{0} \Delta_{f}}\right)
$$

with $P_{i_{f}, i_{t}}^{(n)}[k]$ and $N_{0}$ denoting the transmit power on the $k$ th subcarrier and the noise spectral density, respectively.

Let $\mathbf{B}^{(n)} \in\{0,1\}^{N_{f} \times N_{t}}$ denote the Boolean selection matrix that indicates whether vehicle $n$ uses the corresponding time-frequency block $\left(i_{f}, i_{t}\right) \in \mathcal{F} \times \mathcal{T}$, where $N_{f}=|\mathcal{F}|$ and $N_{t}=|\mathcal{T}|$. In the proposed resource allocation framework, the optimization variables are the Boolean selection vectors of all vehicles $\left\{\mathbf{b}^{(n)}\right\}_{n \in \mathcal{N}}$, where $\mathbf{b}^{(n)} \triangleq \operatorname{vec}\left(\mathbf{B}^{(n)}\right) \in\{0,1\}^{N_{f} N_{t}}$, with $\operatorname{vec}(\cdot)$ representing the matrix vectorization operator stacking the columns on top of each other. Then, the communication objective on the right-hand side of (1) can be expressed as a function of $\mathbf{b}^{(n)}$ as

$$
g^{(n)}\left(\mathbf{b}^{(n)}\right)=\left(\mathbf{r}^{(n)}\right)^{T} \mathbf{b}^{(n)}
$$

where $\mathbf{r}^{(n)} \triangleq \operatorname{vec}\left(\mathbf{R}^{(n)}\right) \in \mathbb{R}^{N_{f} N_{t}}$, with $\mathbf{R}^{(n)} \in \mathbb{R}^{N_{f} \times N_{t}}$ consisting of the elements $R_{i_{f}, i_{t}}^{(n)}$ in (2) for $\left(i_{f}, i_{t}\right) \in \mathcal{F} \times \mathcal{T}$. Equipped with the knowledge of statistical CSIs $\sigma_{i_{f}}^{(n, \mathcal{R}(n))}[k]$ over all subcarriers and transmitter-receiver pairs, the BS can adjust its resource allocation strategy by employing (3) as the communication performance criterion.

\section{Radar PERformance Metric}

\section{A. Radar Signal Model}

For radar performance, we consider the accuracy of the estimation of delay $\tau$ and Doppler $\nu$, for a typical target (specified by a distance $R=c \tau / 2$, velocity $v=c \nu / 2$, and complex channel gain $\alpha$ ). The OFDM radar observation at a given vehicle (dropping the superscript $(n)$ for simplicity) for the block $\left(i_{f}, i_{t}\right)$ can be expressed as ${ }^{1}$

$$
\begin{aligned}
\mathbf{Y}_{i_{f}, i_{t}}=b_{i_{f}, i_{t}} & {\left[\mathbf{P}_{i_{f}, i_{t}} \odot \mathbf{X}_{i_{f}, i_{t}} \odot \alpha\left(\boldsymbol{\Theta}_{i_{f}}\left(\tau_{i_{t}}\right)^{*} \otimes \boldsymbol{\Phi}_{i_{t}}(\nu)^{T}\right)\right.} \\
& \left.+\mathbf{N}_{i_{f}, i_{t}}\right]
\end{aligned}
$$

where

$$
\begin{aligned}
& \boldsymbol{\Theta}_{i_{f}}(\tau) \triangleq e^{j 2 \pi\left(f_{c}+i_{f} W_{\text {block }}\right) \tau}\left[1, e^{j 2 \pi \Delta_{f} \tau}, \ldots, e^{j 2 \pi \Delta_{f}(K-1) \tau}\right]^{T} \\
& \boldsymbol{\Phi}_{i_{t}}(\nu) \triangleq e^{j 2 \pi f_{c} i_{t} T_{\text {block }} \nu}\left[1, e^{j 2 \pi f_{c} T_{\mathrm{s}} \nu}, \ldots, e^{j 2 \pi f_{c}(M-1) T_{\mathrm{s}} \nu}\right]^{T}
\end{aligned}
$$

represent delay and Doppler steering vectors, respectively, $\tau_{i_{t}} \triangleq \tau-\nu i_{t} T_{\text {block }}$ is the delay for the block $\left(i_{f}, i_{t}\right), f_{c}$ is the carrier frequency, $\odot$ and $\otimes$ denote Hadamard and Kronecker product, respectively, $\mathbf{Y}_{i_{f}, i_{t}} \in \mathbb{C}^{K \times M}$ contains the received $M$ OFDM symbols with $K$ subcarriers each, $\mathbf{P}_{i_{f}, i_{t}} \in \mathbb{R}^{K \times M}$ is the matrix of transmit powers on subcarriers with the entries $\mathbf{P}_{i_{f}, i_{t}}^{(n)}[k, m]=\sqrt{P_{i_{f}, i_{t}}^{(n)}[k]}, \forall k, m, \mathbf{X}_{i_{f}, i_{t}} \in \mathbb{C}^{K \times M}$ consists of independent and identically distributed (i.i.d) zeromean, unit-variance complex Gaussian transmit symbols (i.e., $\operatorname{vec}\left(\mathbf{X}_{i_{f}, i_{t}}\right) \sim \mathcal{C N}(\mathbf{0}, \mathbf{I})$ ), and $\mathbf{N}_{i_{f}, i_{t}} \in \mathbb{C}^{K \times M}$ contains i.i.d. additive Gaussian noise components with variance $\sigma^{2}$.

\section{B. Fisher Information Analysis}

From the observations $\mathbf{Y}=\left\{\mathbf{Y}_{i_{f}, i_{t}}\right\}_{\left(i_{f}, i_{t}\right) \in \mathcal{F} \times \mathcal{T}}$, the modified Fisher information matrix (MFIM) [11] will be used to evaluate the accuracy of estimation of $\tau$ and $\nu$ since the transmitted symbols $\mathbf{X}_{i_{f}, i_{t}}$ are not deterministic. The parameters of interest for the MFIM are given by $\boldsymbol{\eta}=\left[\tau, \nu, \alpha_{\mathrm{R}}, \alpha_{\mathrm{I}}\right]^{T}$, where $\alpha_{\mathrm{R}} \triangleq \Re\{\alpha\}$ and $\alpha_{\mathrm{I}} \triangleq \Im\{\alpha\}$. The $(i, j)$ th element of the $4 \times 4$ MFIM can be written as [11]

$$
\begin{aligned}
& \mathbf{J}_{\boldsymbol{\eta}}[i, j] \\
& =\mathbb{E}_{\mathbf{X}}\left\{\mathbb{E}_{\mathbf{Y} \mid \mathbf{X}}\left\{\frac{\partial \log \Lambda(\mathbf{Y} \mid \mathbf{X}, \boldsymbol{\eta})}{\partial \eta[i]} \frac{\partial \log \Lambda(\mathbf{Y} \mid \mathbf{X}, \boldsymbol{\eta})}{\partial \eta[j]}\right\}\right\}
\end{aligned}
$$

\footnotetext{
${ }^{1}$ Derivations are omitted due to space limitations.
} 
where $\log \Lambda(\mathbf{Y} \mid \mathbf{X}, \boldsymbol{\eta})$ is the $\log$-likelihood function of the observations in (4) with $\mathbf{X}=\left\{\mathbf{X}_{i_{f}, i_{t}}\right\}_{\left(i_{f}, i_{t}\right) \in \mathcal{F} \times \mathcal{T}}$. After algebraic manipulations on (5), we obtain the MFIM as

$$
\mathbf{J}_{\boldsymbol{\eta}}=\left[\begin{array}{cc}
\left(\mathbf{I}_{2} \otimes \mathbf{b}\right)^{T} \mathbf{J}_{11} & \left(\mathbf{I}_{2} \otimes \mathbf{b}\right)^{T} \mathbf{J}_{12} \\
\mathbf{J}_{12}^{T}\left(\mathbf{I}_{2} \otimes \mathbf{b}\right) & \left(\mathbf{I}_{2} \otimes \mathbf{b}\right)^{T} \mathbf{J}_{22}
\end{array}\right] \in \mathbb{R}^{4 \times 4}
$$

where $\mathbf{I}_{N}$ is the $N \times N$ identity matrix, and the submatrices $\mathbf{J}_{11}, \mathbf{J}_{12}$ and $\mathbf{J}_{22}$ are given in Appendix A. Applying the matrix inversion lemma to (6), we obtain $\left[\mathbf{J}_{\boldsymbol{\eta}}^{-1}\right]_{1: 2,1: 2}=$ $(\mathbf{G}(\mathbf{b}))^{-1}$, where

$$
\begin{aligned}
\mathbf{G}(\mathbf{b}) \triangleq & \left(\mathbf{I}_{2} \otimes \mathbf{b}\right)^{T} \mathbf{J}_{11} \\
& -\left(\mathbf{I}_{2} \otimes \mathbf{b}\right)^{T} \mathbf{J}_{12}\left(\left(\mathbf{I}_{2} \otimes \mathbf{b}\right)^{T} \mathbf{J}_{22}\right)^{-1} \mathbf{J}_{12}^{T}\left(\mathbf{I}_{2} \otimes \mathbf{b}\right)
\end{aligned}
$$

represents the equivalent MFIM for delay and Doppler parameters. To optimize the delay-Doppler estimation performance of radar, we use the modified Cramér-Rao bound (MCRB) on the covariance matrix of delay and Doppler estimates as our design objective. The MCRB matrix can be obtained as the inverse of the MFIM in (7) [11]. Hence, delay and Doppler estimation variances can be expressed, respectively, as

$$
f_{\tau}(\mathbf{b})=\left[(\mathbf{G}(\mathbf{b}))^{-1}\right]_{1,1}, f_{\nu}(\mathbf{b})=\left[(\mathbf{G}(\mathbf{b}))^{-1}\right]_{2,2}
$$

\section{Joint Radar COMmunications Based Resource ALLOCATION}

\section{A. Problem Statement}

Our aim is to optimize the joint radar communications performance of the vehicular network by using the sum SE in (3) and the CRBs in (8) as our design objectives. For characterization of performance trade-off between the two functionalities, the problem of resource allocation can be formulated as a weighted optimization of radar delay-Doppler estimation performance and communication SE as follows:

$$
\begin{array}{cc}
\underset{\left\{\mathbf{b}^{(n)}\right\}_{n \in \mathcal{N}}}{\operatorname{minimize}} & w_{\text {rad }} \sum_{n \in \mathcal{N}}\left[w_{\tau} f_{\tau}^{(n)}\left(\mathbf{b}^{(n)}\right)+\left(1-w_{\tau}\right) f_{\nu}^{(n)}\left(\mathbf{b}^{(n)}\right)\right] \\
& -\left(1-w_{\text {rad }}\right) \sum_{n \in \mathcal{N}} g^{(n)}\left(\mathbf{b}^{(n)}\right) \\
\text { subject to } & \mathbf{b}^{(n)} \in\{0,1\}{ }^{N_{f} N_{t}}, \forall n \in \mathcal{N} \\
& \mathbf{e}_{i_{t}}^{T} \mathbf{b}^{(n)} \leq 1, \forall n \in \mathcal{N}, \forall i_{t} \in \mathcal{T} \\
& \sum_{n \in \mathcal{N}} \mathbf{b}^{(n)} \preceq \mathbf{1}_{N_{f} N_{t}} \\
& \mathbf{1}_{N_{f} N_{t}}^{T} \mathbf{b}^{(n)} \leq \kappa_{n}, \forall n \in \mathcal{N}
\end{array}
$$

where $f_{\tau}^{(n)}\left(\mathbf{b}^{(n)}\right)$ and $f_{\nu}^{(n)}\left(\mathbf{b}^{(n)}\right)$ are the vehicle-specific versions of (8), $\mathbf{e}_{i_{t}} \in \mathbb{R}^{N_{f} N_{t}}$ is an all 0's vector except 1's at the indices between $i_{t} N_{f}+1$ and $\left(i_{t}+1\right) N_{f}, \mathbf{1}_{N}$ is an all 1's vector of size $N$, and $\kappa_{n}$ is the upper bound on the number of blocks used by vehicle $n$, which is due to (i) power consumption requirements of vehicles and (ii) efficient usage of spectrum.

In (9), $w_{\tau}$ and $1-w_{\tau}$ denote the preset weightings of delay and Doppler estimation accuracies, while $w_{\text {rad }}$ and $1-w_{\text {rad }}$ are weighting factors for radar and communication objectives, with $0 \leq w_{\tau}, w_{\text {rad }} \leq 1$. In addition, the constraint (9c) guarantees that each vehicle occupies at most one timefrequency resource block over a block duration (imposed by ADC bandwidth), while the constraint (9d) ensures orthogonality of vehicles in the time-frequency domain. The problem (9) is a nonlinear integer programming problem with high computational complexity. In the following, we propose to relax the Boolean constraint in $(9 b)$ to obtain an efficiently solvable convex program.

\section{B. Convexification of (9) via Boolean Relaxation}

By introducing the slack variables $\beta_{\tau, n}, \beta_{\nu, n}$ and $\mathbf{A}^{(n)} \in$ $\mathbb{S}_{+}^{2}$, where $\mathbb{S}_{+}^{N}$ is the set of $N \times N$ symmetric positive semidefinite matrices, (9) can be rewritten as follows:

$$
\begin{array}{ll}
\underset{\substack{\left\{\mathbf{b}^{(n)}, \mathbf{A}^{(n)}, \beta_{\tau, n}, \beta_{\nu, n}\right\}_{n \in \mathcal{N}}}}{\operatorname{minimize}} & w_{\mathrm{rad}} \sum_{n \in \mathcal{N}}\left[w_{\tau} \beta_{\tau, n}+\left(1-w_{\tau}\right) \beta_{\nu, n}\right] \\
& -\left(1-w_{\mathrm{rad}}\right) \sum_{n \in \mathcal{N}}\left(\mathbf{r}^{(n)}\right)^{T} \mathbf{b}^{(n)} \\
\text { subject to } & \mathbf{G}^{(n)}\left(\mathbf{b}^{(n)}\right) \succeq\left[\mathbf{A}^{(n)}\right]^{-1}, \forall n \in \mathcal{N} \\
& \operatorname{diag}\left(\mathbf{A}^{(n)}\right) \preceq\left[\beta_{\tau, n}, \beta_{\nu, n}\right], \forall n \in \mathcal{N} \\
& (9 \mathrm{~b})-(9 \mathrm{e})
\end{array}
$$

where $\mathbf{G}^{(n)}\left(\mathbf{b}^{(n)}\right)$ is the vehicle-specific version of (7) and $\operatorname{diag}(\cdot)$ represents the diagonal entries of a matrix. Based on the definition of $\mathbf{G}^{(n)}\left(\mathbf{b}^{(n)}\right)$ in (7) and the Schur complement properties [12, Ch. A.5.5], the constraint (10b) can be equivalently reformulated as

$$
\left[\begin{array}{cc}
\left(\mathbf{I}_{2} \otimes \mathbf{b}^{(n)}\right)^{T} \mathbf{J}_{11}^{(n)}-\mathbf{C}^{(n)} & \mathbf{I}_{2} \\
\mathbf{I}_{2} & \mathbf{A}^{(n)}
\end{array}\right] \succeq 0, \forall n \in \mathcal{N}
$$

$$
\left[\begin{array}{cc}
\mathbf{C}^{(n)} & \left(\mathbf{I}_{2} \otimes \mathbf{b}^{(n)}\right)^{T} \mathbf{J}_{12}^{(n)} \\
\left(\mathbf{J}_{12}^{(n)}\right)^{T}\left(\mathbf{I}_{2} \otimes \mathbf{b}^{(n)}\right) & \left(\mathbf{I}_{2} \otimes \mathbf{b}^{(n)}\right)^{T} \mathbf{J}_{22}^{(n)}
\end{array}\right] \succeq 0, \forall n \in \mathcal{N}
$$

where we introduce the slack variables $\mathbf{C}^{(n)} \in \mathbb{S}_{+}^{2}$. We note that the equivalent problem then has a linear objective, Boolean constraints (9b), linear inequality constraints (9c)(9e) and (10c), and linear matrix inequality (LMI) constraints (11). Via Boolean relaxation of (9b) to its convex hull $[0,1]^{N_{f} N_{t}}$, the problem (10a) can be recast in a relaxed form that turns out to be an SDP and thus can be solved efficiently using off-the-shelf convex optimization tools [13]. With this relaxation, the continuous variables $\mathbf{b}^{(n)}$ can now be interpreted as time-sharing factors [14] such that vehicle $n$ uses the block $\left(i_{f}, i_{t}\right)$ for $M b_{i_{f}, i_{t}}^{(n)}$ symbols out of $M$ symbols $^{2}$.

\footnotetext{
${ }^{2}$ This physical interpretation holds true under the assumption that the individual summands of MFIM elements in (12) and (13) have negligible variation across time dimension (i.e., symbol dimension $m$ ) within a timefrequency block compared to variation between different blocks.
} 


\section{REsults}

In this part, we evaluate the performance of the proposed joint radar communications resource allocation approach via simulations. We consider a scenario consisting of three vehicles with 2-D positions $\mathbf{x}_{1}=\left[\begin{array}{ll}0 & 0\end{array}\right] \mathrm{m}, \mathbf{x}_{2}=\left[\begin{array}{ll}10 & 0\end{array}\right] \mathrm{m}$ and $\mathbf{x}_{3}=\left[\begin{array}{ll}25 & 3\end{array}\right] \mathrm{m}$, sharing a time-frequency resource with $f_{c}=60 \mathrm{GHz}, W_{\mathrm{S}}=500 \mathrm{MHz}$ and $T_{\text {frame }}=20 \mathrm{~ms}$. We assume the existence of three communication links with transmit-receive pairs $\{(1,2),(3,1),(2,3)\}$. The OFDM system parameters are set as $W_{\text {block }}=50 \mathrm{MHz}, T_{\text {block }}=2 \mathrm{~ms}$, $\Delta_{f}=500 \mathrm{KHz}, T=2 \mu \mathrm{s}, T_{\mathrm{cp}}=0.5 \mu \mathrm{s}, K=100$, $M=800, N_{f}=N_{t}=10$. The transmit powers $P_{i_{f}, i_{t}}^{(n)}[k]$ are taken to be uniform across all subcarriers, time-frequency blocks and vehicles, and the total power over a block is set to $-20 \mathrm{dBm}$, while the noise spectral density $N_{0}$ is taken as $4.0038 \times 10^{-21} \mathrm{~W} / \mathrm{Hz}$. We simulate the radar channel gains according to radar range equation [15, Ch. 2.2] with typical vehicle RCS values (e.g., $[0,5] \mathrm{dBsm})$ and $10 \mathrm{dBi}$ antenna gains. The statistical CSIs $\sigma_{i_{f}}^{(n, \mathcal{R}(n))}[k]$ are assumed to be equal across subcarriers and generated based on the free-space path loss model [10, Ch. 2.3]. For the constraint (9e), we set $\kappa_{n}=5, \forall n \in \mathcal{N}$.

Fig. 2 illustrates the radar-communication performance trade-off curves corresponding to the solution of the relaxed version of (10). By tuning the trade-off parameter $w_{\text {rad }}$, the vehicular network can transition between radar-optimal $\left(w_{\text {rad }}=1\right)$ and communication-optimal $\left(w_{\text {rad }}=0\right)$ operation regimes, depending on system requirements. As $w_{\text {rad }}$ approaches 0 , all trade-off curves asymptotically converge to the maximum SE that can be achieved in radar-free operation. In addition, range accuracy is more sensitive to changes in SE than velocity accuracy since the expression (2) varies only over frequency due to uniform subcarrier powers and time-invariant channel statistics over a frame duration $T_{\text {frame }}$. Moreover, it is observed that better Pareto-optimal solutions can be achieved with higher RCS values, as expected. This suggests, for example, that data rates can be increased in an environment with large RCS objects without changing radar accuracy constraints.

To investigate resource allocation results in the timefrequency domain, Fig. 3 demonstrates the optimal timesharing factors $\mathbf{b}^{(n)}$ for different weighting factors $w_{\text {rad }}$ and $w_{\tau}$, along with the $\operatorname{SE} \mathbf{r}^{(n)}$ of the three links over the frequency blocks. First, Fig. 3(b) shows the communicationoptimal allocation, which complies with the SE plots in Fig. 3(a) (i.e., each link is assigned the frequency block with the highest data rate). Second, Fig. 3(c), Fig. 3(d) and Fig. 3(e) illustrate radar-optimal time-frequency allocations. It is observed that the delay-optimal solution focuses all the available power on the edges of the frequency spectrum to maximize the root mean square (RMS) bandwidth, while the Doppler-optimal solution utilizes the edges of the time window to maximize the RMS envelope [16]. On the other hand, the delay-Doppler-optimal allocation occupies the corners of the available time-frequency region as a compromise between the

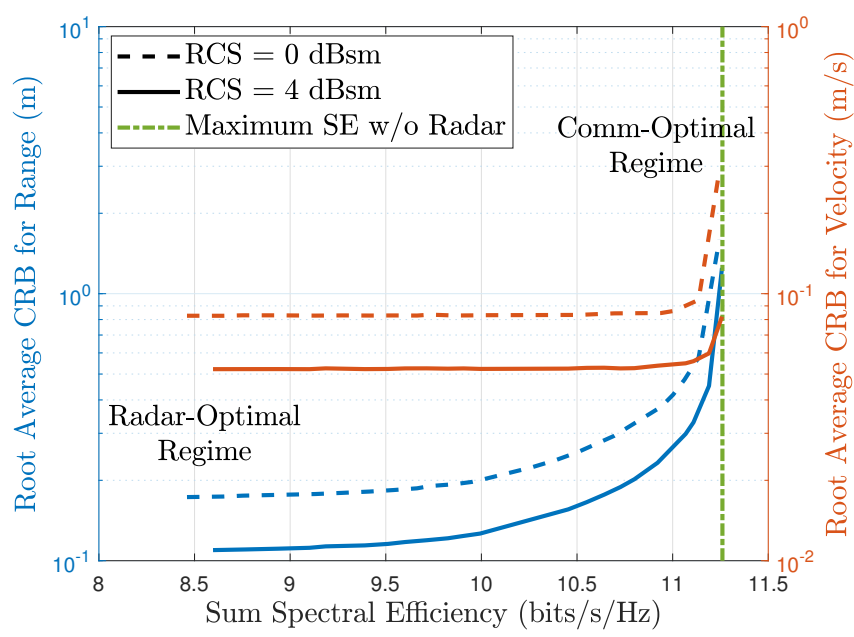

Fig. 2. Radar-communication trade-off curves for multiple target RCS values along with the asymptotic SE line in radar-free operation, where root average CRBs on range and velocity estimation (with $w_{\tau}=0.5$ ) are plotted against sum SE as $w_{\text {rad }}$ varies over the interval $[0,1]$ in the relaxed version of (10). Due to time-invariant, frequency-selective channel statistics and uniform subcarrier powers, the trade-off on the radar side is mainly related to the accuracy of range rather than that of velocity.

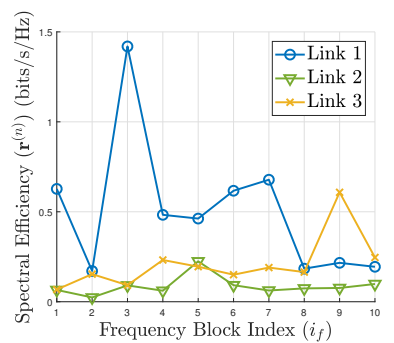

(a)

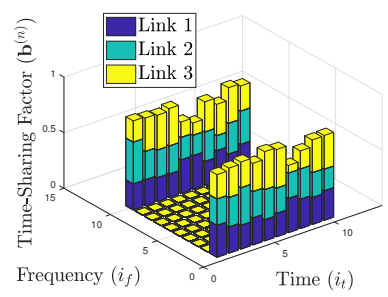

(c)

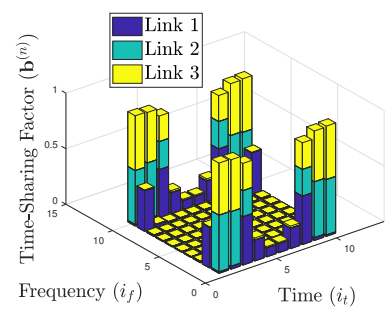

(e)

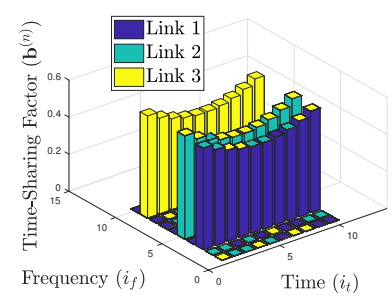

(b)

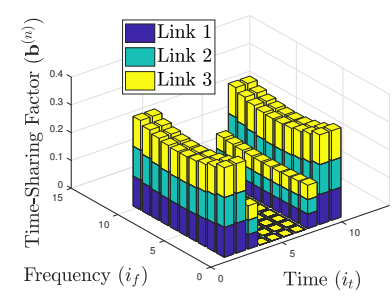

(d)

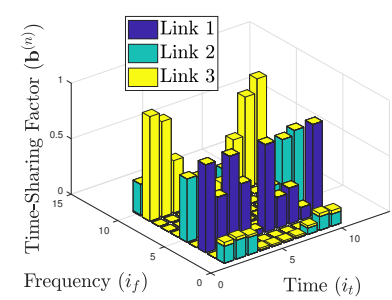

(f)
Fig. 3. Optimal time-frequency resource allocation results obtained from the solution of the relaxed version of (10). (a) Time-invariant block SE values $\mathbf{r}^{(n)}$ in (3) over different frequency blocks. (b) Communication-optimal resource allocation with $w_{\text {rad }}=0$. (c) Radar delay-optimal resource allocation with $w_{\text {rad }}=1$ and $w_{\tau}=1$. (d) Radar Doppler-optimal resource allocation with $w_{\mathrm{rad}}=1$ and $w_{\tau}=0$. (e) Radar delay-Doppler-optimal resource allocation with $w_{\text {rad }}=1$ and $w_{\tau}=0.5$. (f) Weighted radar-communication resource allocation with $w_{\text {rad }}=0.8$ and $w_{\tau}=0.5$. 
delay- and Doppler-optimal solutions. Finally, Fig. 3(f) shows the weighted radar-communication design, which reflects both delay-Doppler optimality in Fig. 3(e) and link qualities in Fig. 3(a).

\section{CONCLUSIONS}

We consider a centralized V2V time-frequency resource allocation scheme for stepped-carrier OFDM based vehicular networks, where each vehicle is assigned a number of OFDM time-frequency resource blocks with the aim of improved overall joint radar communications performance. To investigate performance trade-offs, a nonlinear integer programming problem with Boolean resource selection variables is formulated to optimize weighted average of radar estimation performance and communication spectral efficiency. Simulation results reveal the compromise between the two tasks, indicating Pareto-optimal solutions for different target RCS values. In addition, the interplay between delay-optimal, Doppleroptimal and communication-optimal designs is illustrated in the time-frequency domain. We plan to extend this study by incorporating downlink joint radar communications into the existing resource allocation problem.

\section{ACKNOWLEDGMENTS}

This work is supported by Marie Curie Individual Fellowships Grant 745706 (GreenLoc) and Vinnova grant 201801929.

\section{APPENDIX A \\ ELEMENTS OF THE MODIFIED FISHER INFORMATION MATRIX}

We define, for $i_{f} \in \mathcal{F}$ and $i_{t} \in \mathcal{T}$,

$$
\begin{aligned}
& \vartheta\left[i_{f}+N_{f} i_{t}\right] \\
& =-j 2 \pi \sum_{m=0}^{M-1} \sum_{k=0}^{K-1} P_{i_{f}, i_{t}}[k, m]\left(f_{c}+i_{f} W_{\text {block }}+k \Delta_{f}\right) \\
& \widetilde{\vartheta}\left[i_{f}+N_{f} i_{t}\right] \\
& =4 \pi^{2} \sum_{m=0}^{M-1} \sum_{k=0}^{K-1} P_{i_{f}, i_{t}}[k, m]\left(f_{c}+i_{f} W_{\text {block }}+k \Delta_{f}\right)^{2} \\
& \varphi\left[i_{f}+N_{f} i_{t}\right] \\
& =-j 2 \pi \sum_{m=0}^{M-1} \sum_{k=0}^{K-1} P_{i_{f}, i_{t}}[k, m] f_{c}\left(i_{t} T_{\text {block }}+(m+1) T_{\mathrm{s}}\right) \\
& \widetilde{\varphi}\left[i_{f}+N_{f} i_{t}\right] \\
& =4 \pi^{2} \sum_{m=0}^{M-1} \sum_{k=0}^{K-1} P_{i_{f}, i_{t}}[k, m] f_{c}^{2}\left(i_{t} T_{\text {block }}+(m+1) T_{\mathrm{s}}\right)^{2} \\
& \xi\left[i_{f}+N_{f} i_{t}\right] \\
& =4 \pi^{2} \sum_{m=0}^{M-1} \sum_{k=0}^{K-1} P_{i_{f}, i_{t}}[k, m] f_{c}\left(f_{c}+i_{f} W_{\text {block }}+k \Delta_{f}\right) \\
& \quad \times\left(i_{t} T_{\text {block }}+(m+1) T_{\mathrm{s}}\right)
\end{aligned}
$$

$$
\text { and } p\left[i_{f}+N_{f} i_{t}\right]=\sum_{m=0}^{M-1} \sum_{k=0}^{K-1} P_{i_{f}, i_{t}}[k, m] .
$$

Then, the submatrices constituting the MFIM in (6) are derived as follows:

$$
\begin{aligned}
& \mathbf{J}_{11}=\frac{2|\alpha|^{2}}{\sigma^{2}}\left[\begin{array}{cc}
\widetilde{\boldsymbol{\vartheta}} & \boldsymbol{\xi} \\
\boldsymbol{\xi} & \widetilde{\boldsymbol{\varphi}}
\end{array}\right] \in \mathbb{R}^{2 N_{f} N_{t} \times 2} \\
& \mathbf{J}_{12}=\frac{2}{\sigma^{2}}\left[\begin{array}{cc}
\Re\left\{\alpha^{*} \boldsymbol{\vartheta}\right\} & -\Im\left\{\alpha^{*} \boldsymbol{\vartheta}\right\} \\
\Re\left\{\alpha^{*} \boldsymbol{\varphi}\right\} & -\Im\left\{\alpha^{*} \boldsymbol{\varphi}\right\}
\end{array}\right] \in \mathbb{R}^{2 N_{f} N_{t} \times 2} \\
& \mathbf{J}_{22}=\frac{2}{\sigma^{2}}\left[\begin{array}{cc}
\mathbf{p} & \mathbf{0}_{N_{f} N_{t}} \\
\mathbf{0}_{N_{f} N_{t}} & \mathbf{p}
\end{array}\right] \in \mathbb{R}^{2 N_{f} N_{t} \times 2}
\end{aligned}
$$

where $\mathbf{0}_{N}$ is an all 0 's vector of size $N$.

\section{REFERENCES}

[1] A. R. Chiriyath, B. Paul, and D. W. Bliss, "Radar-communications convergence: Coexistence, cooperation, and co-design," IEEE Transactions on Cognitive Communications and Networking, vol. 3, no. 1, pp. 1-12, March 2017.

[2] S. M. Patole, M. Torlak, D. Wang, and M. Ali, "Automotive radars: A review of signal processing techniques," IEEE Signal Processing Magazine, vol. 34, no. 2, pp. 22-35, March 2017.

[3] C. Aydogdu, G. K. Carvajal, O. Eriksson, H. Hellsten, H. Herbertsson, M. F. Keskin, E. Nilsson, M. Rydström, K. Vanäs, and H. Wymeersch, "Radar Interference Mitigation for Automated Driving," arXiv e-prints, p. arXiv:1909.09441, Sep 2019.

[4] C. Sturm and W. Wiesbeck, "Waveform design and signal processing aspects for fusion of wireless communications and radar sensing," Proceedings of the IEEE, vol. 99, no. 7, pp. 1236-1259, July 2011.

[5] C. D. Ozkaptan, E. Ekici, O. Altintas, and C. Wang, "OFDM pilot-based radar for joint vehicular communication and radar systems," in 2018 IEEE Vehicular Networking Conference (VNC), Dec 2018, pp. 1-8.

[6] M. Bic and V. Koivunen, "Multicarrier radar-communications waveform design for RF convergence and coexistence," in ICASSP 2019 - 2019 IEEE International Conference on Acoustics, Speech and Signal Processing (ICASSP), May 2019, pp. 7780-7784.

[7] Y. Liu, G. Liao, J. Xu, Z. Yang, and Y. Zhang, "Adaptive OFDM integrated radar and communications waveform design based on information theory," IEEE Communications Letters, vol. 21, no. 10, pp. 2174-2177, Oct 2017.

[8] Y. Liu, G. Liao, Z. Yang, and J. Xu, "Multiobjective optimal waveform design for OFDM integrated radar and communication systems," Signal Processing, vol. 141, pp. 331-342, 2017.

[9] B. Schweizer, C. Knill, D. Schindler, and C. Waldschmidt, "Steppedcarrier OFDM-radar processing scheme to retrieve high-resolution range-velocity profile at low sampling rate," IEEE Transactions on Microwave Theory and Techniques, vol. 66, no. 3, pp. 1610-1618, March 2018.

[10] A. Goldsmith, Wireless Communications. Cambridge University Press, 2005.

[11] A. N. D'Andrea, U. Mengali, and R. Reggiannini, "The modified Cramér-Rao bound and its application to synchronization problems," IEEE Transactions on Communications, vol. 42, no. 234, pp. 13911399, February 1994.

[12] S. Boyd and L. Vandenberghe, Convex Optimization. Cambridge university press, 2004.

[13] M. Grant and S. Boyd, "CVX: Matlab software for disciplined convex programming, version 2.1," http://cvxr.com/cvx, Mar. 2014.

[14] C. Y. Wong, R. S. Cheng, K. B. Lataief, and R. D. Murch, "Multiuser OFDM with adaptive subcarrier, bit, and power allocation," IEEE Journal on Selected Areas in Communications, vol. 17, no. 10, pp. 17471758, 1999.

[15] M. A. Richards, Fundamentals of Radar Signal Processing. Tata McGraw-Hill Education, 2005.

[16] B. Paul and D. W. Bliss, "Extending joint radar-communications bounds for FMCW radar with Doppler estimation," in 2015 IEEE Radar Conference (RadarCon), May 2015, pp. 0089-0094. 